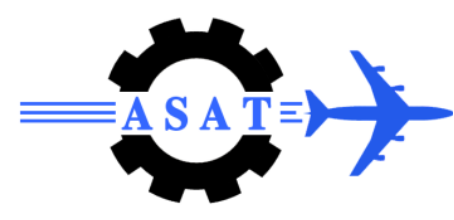

\title{
Focused ISAR Imaging of Rotating Target in Far-Field Compact Range Anechoic Chamber
}

\author{
Shahida G. Qadir ${ }^{*}$, Fan Yanyu ${ }^{\dagger}$, Ahmed S. Amein ${ }^{\ddagger}$, Fathy M. Ahmed ${ }^{\S}$
}

\begin{abstract}
A case study of a modified Inverse Synthetic Aperture Radar (ISAR) imaging technique for different target geometries in the Far Field RCS measurement Compact Range is presented. The processing applied to the experimental data is a combination of two high resolution processes: dev of High Range Resolution profile (HRR) in the down range using stepped frequency waveform covering a total band of $4 \mathrm{GHz}$, and the development of high resolution profile in cross-range is achieved using ISAR technique. The manuscript shows the use of Polar reformatting algorithm with Shannon reconstruction technique for correcting migration through resolution cells to obtain a better focused image of rotating targets.
\end{abstract}

Keywords: FM, ISAR, Polar, Radar Imaging, RCS measurement

\section{Introduction}

Radar imaging and multi-dimensional radar systems have become widely used in many areas, both military and commercial. This is due to the advent of more advanced waveform generation and signal processing techniques.

The basic principle of Inverse Synthetic Aperture Radar (ISAR) imaging is to coherently collect the wideband echo signals that are produced as the target rotates to present a change in viewing angle to the radar The resultant ISAR image produced after required processing is a representation of the spatial distribution of the reflectivity of a target (Radar Cross Section). The target's reflectivity is usually mapped onto a down-range versus cross-range plane, and is viewed as a radar image of the target [1, 2, $3 \& 4]$.

\section{Compact Range Test Setup \& Data Acquisition}

In order to examine the proposed ISAR imaging technique, experiments are conducted in FarField Compact Range Anechoic chamber. The Compact range used is employed with dual parabolic reflectors to focus the RF energy into a plane wave within a much shorter distance than would normally be required based on the spherical wave-front spreading [5].

\footnotetext{
School of Electronics \& Information Processing, Northwestern Polytechnical University, Xi'an, Shaanxi, China, shahida_qadir@yahoo.com

${ }^{\dagger}$ Professor, School of Electronics \& Information Processing, Northwestern Polytechnical University, Xi'an, Shaanxi, China, fan_yangyu@ sina.com

* Egyptian Armed Forces, asamein@gmail.com

${ }^{\S}$ Egyptian Armed Forces, fkader2003@yahoo.com
} 
The imaging process includes the following main steps:

1) The target under test (spheres, trihedral corner reflectors) is irradiated with a plane electromagnetic wave of variable frequency $f$ covering a band of $4 \mathrm{GHz}$. The target is mounted on a positioner with axis of rotation perpendicular to the direction of propagation of the incoming wave (Fig. 1).

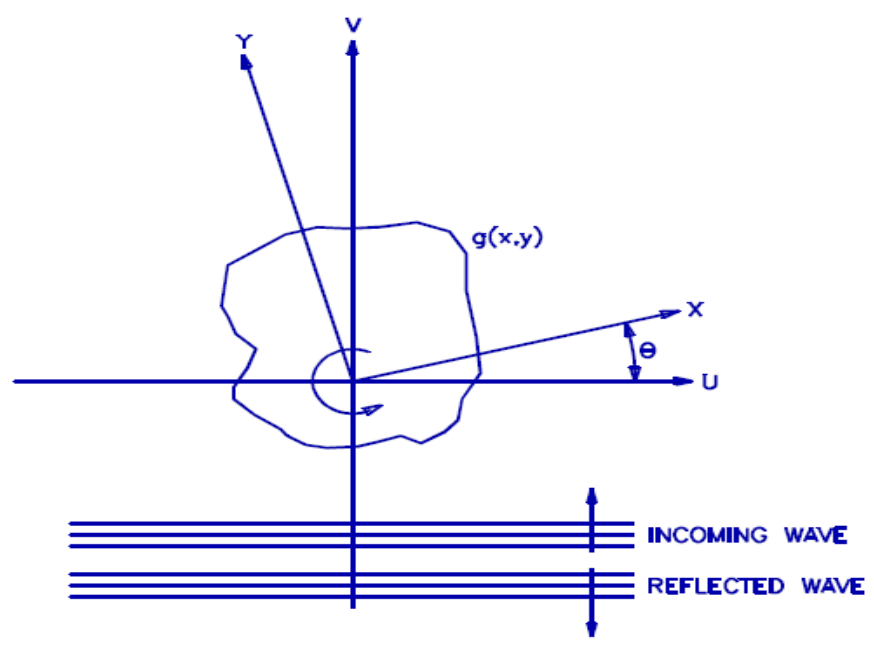

Fig. 1 Rotating target illumination by plane waveform

2) The wave reflected by the target is measured in amplitude and phase by compact range data acquisitioning system. The echo signal from a rotating target is given by $[4,5]$ as follows:

$$
\begin{aligned}
\mathrm{S}(\mathrm{f}, \theta)=\sum_{\mathrm{k}=1}^{\mathrm{L}} \mathrm{a}_{\mathrm{k}} \exp \left(-\mathrm{j} \frac{4 \pi \mathrm{f}}{\mathrm{c}}\left(\mathrm{x}_{\mathrm{k}} \cos \theta+\mathrm{y}_{\mathrm{k}} \sin \theta\right)\right)+\mathrm{u}(\mathrm{f}, \theta) \\
S(f, \theta)=\sum_{k=1}^{L} a_{k} \exp \left(-j \frac{4 \pi f}{c}\left(x_{k} \cos (\theta)+y_{k} \sin (\theta)\right)\right)+u(f, \theta)
\end{aligned}
$$

where,

$$
\begin{array}{ll}
c & \text { the speed of light waves, } \\
f & \text { transmitted frequency, } \\
\theta & \text { rotating angle } \\
x, y & \text { target position }
\end{array}
$$

3) The received Radar Cross Section (RCS) data uniformly sampled in the frequency aspect domain $(f, \theta)$ does not correspond to the uniformly sampled data in the spatial frequency domain $\left(f_{x}, f_{y}\right)$ due to non-linear relationship given by $\left(f_{x}=\frac{2 f}{c} \sin \theta, f_{y}=\frac{2 f}{c} \cos \theta\right)$. 


\section{Applied Unfocused and Focused Imaging Methods}

\section{a. Unfocused Imaging}

Narrow angle approximated spatial domain $\left(\mathrm{f}_{\mathrm{x}} \approx \frac{2 \mathrm{f}}{\mathrm{c}} \theta, \mathrm{f}_{\mathrm{y}} \approx \frac{2 \mathrm{f}}{\mathrm{c}}\right)$ approximately makes a Cartesian grid without use of interpolation. The required processing to obtain $\mathrm{s}(\mathrm{x}, \mathrm{y})$ from $\mathrm{S}(\mathrm{f}, \theta)$ is 2D-FFT. Due to the approximations made, the resulting image will not be perfect due to Motion Through Resolution Cell (MTRC) [6, 7 \& 8]. Figure 2 depicts the phenomenon of Migration Through Range Resolution (MTRRC) and Migration Through Cross Range Resolution Cell (MTCRRC) for a large rotating target, which occurs if target size exceeds the following dimensions $[4,5,7]$;

Maximum Dimension in Down-range: $D_{r, \max }=\frac{c}{f_{o} \Theta^{2}}$

Maximum Dimension in Cross-range: $\mathrm{D}_{\mathrm{c}, \max }=\frac{\mathrm{c}}{\mathrm{B} \Theta}$

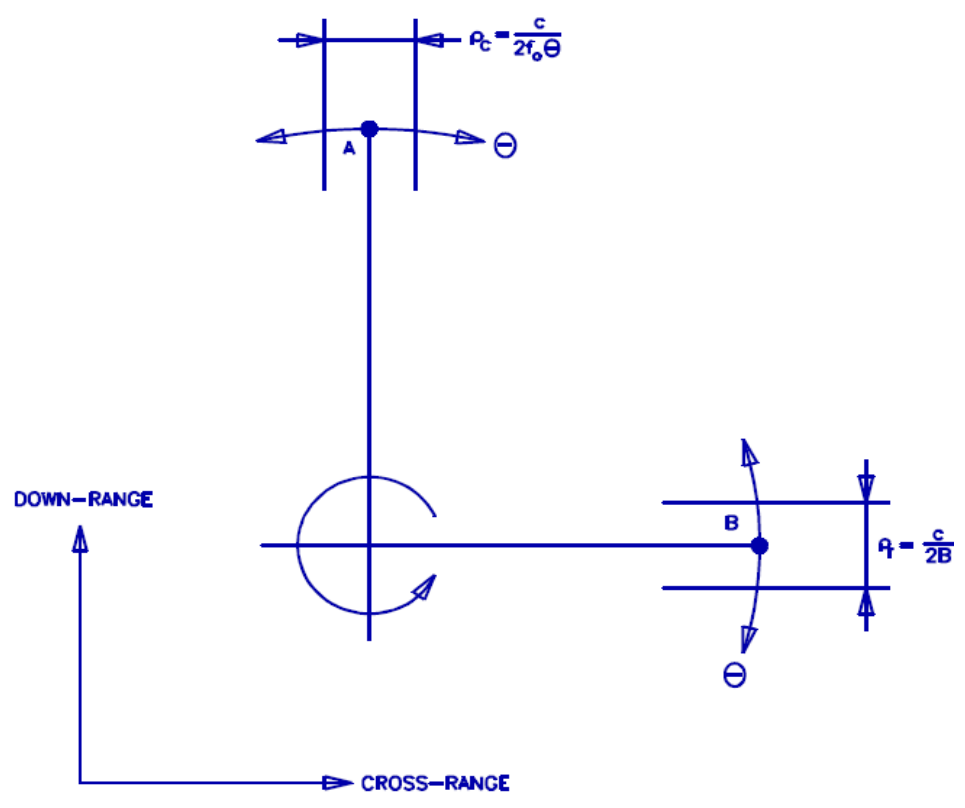

Fig. 2 MTRC phenomenon

\section{b. Focused Imaging}

Data uniformity in spatial frequency domain $\left(f_{x}, f_{y}\right)$ is essential for application of Fast Fourier Transform algorithm or high resolution PSD estimators. Therefore, in order to generate a focused ISAR image, the received data is converted from polar-Formatted samples to Cartesian-Formatted samples with uniform spatial frequency sampling spacing using Shannon reconstruction technique $[8,9]$. The technique is based on sin function interpolation as given below;

$$
f(x)=\sum_{x} s(x) \sin c\left(\frac{x-x_{n}}{\Delta}\right)
$$


where $x_{n}$ is the amplitude of the available samples and $\Delta$ is the sampling space. 1-D mathematical model (Eq. (3)) is used to interpolate data in first in down-range and then in cross-range in order to convert Polar grid data into Cartesian grid data. 2D FFT is applied on Cartesian grid data that compresses the data in range and azimuth to achieve the desired resolution and focused image. FFT being computationally efficient is the first choice in real time radar imaging applications.

\section{Experimental Results and Discussion}

The following three tests are conducted in compact range while the results are summarized in Table 1.

Table 1 Tests $(1,2,3)$ Result Summary

\begin{tabular}{|c|c|c|c|}
\hline \multirow{2}{*}{ Parameters } & \multicolumn{3}{|c|}{ Test Type } \\
\hline & Two spheres & 4 corner Reflectors & 5 Corner reflectors \\
\hline Bandwidth (GHz) & 4 & 4 & 4 \\
\hline Aspect Angle Span & $16^{\circ}$ & $60^{\circ}$ & $60^{\circ}$ \\
\hline Range Resolution (cm) & 3.75 & 3.75 & 3.75 \\
\hline Cross Range Resolution (cm) & 3.25 & 0.89 & 0.89 \\
\hline $\begin{array}{l}\text { Target Size Limits } \\
D_{r, \max } \times D_{c, \max }(\mathrm{cm})\end{array}$ & $24 \times 27$ & $2 \times 7$ & $2 \times 7$ \\
\hline $\begin{array}{l}\text { Actual Target Area } \\
\left(D_{r} \times D_{c}\right)(\mathbf{c m})\end{array}$ & $5 \times 34$ & $40 \times 70$ & $50 \times 50$ \\
\hline MTRRC & 2 cells & 10 cells & 7 cells \\
\hline MTCRRC & 0 cells & 24 cells & 30 cells \\
\hline $\begin{array}{l}\text { Image Produced by Narrow- } \\
\text { Angle Approximation } \\
\text { Approach }\end{array}$ & $\begin{array}{l}\text { Reasonably } \\
\text { Focused }\end{array}$ & Unfocused & $\begin{array}{l}\text { CR5 is well focused, } \\
\text { CR(1,2,3,\&4) are } \\
\text { unfocused }\end{array}$ \\
\hline $\begin{array}{l}\text { Image Produced by Polar } \\
\text { Reformatting using Shannon } \\
\text { Reconstruction }\end{array}$ & $\begin{array}{l}\text { Both spheres } \\
\text { are Highly } \\
\text { Focused }\end{array}$ & $\begin{array}{l}\text { All corner reflectors } \\
\text { are Well Focused }\end{array}$ & $\begin{array}{c}\text { CR }(1,2 \& 5) \text { are well } \\
\text { focused while } \\
\text { CR }(3,4) \text { are still } \\
\text { Blurred }\end{array}$ \\
\hline
\end{tabular}

Test 1: Two Spheres:

In this test two Spheres of diameter $3 \mathrm{~cm}$ and $5 \mathrm{~cm}$ respectively are placed in same range cell and are separated in cross range by $30 \mathrm{~cm}$. The contour plots of resultant images are shown in Fig. 3. Due to negligible MTRRC and 0 MTCRRC, image obtained by narrow angle approximation and Shannon reconstruction are of almost same quality. 

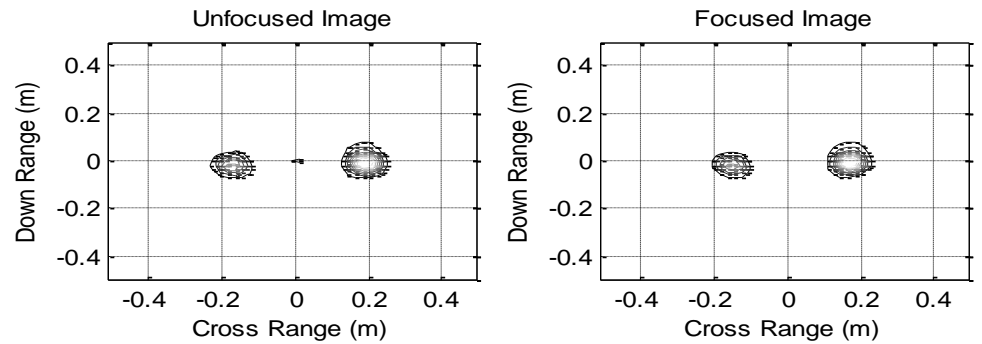

Fig. 3 Target geometry with two spheres

\section{Test 2: Four Trihedral Corner Reflectors}

In this experiment four trihedral corner reflectors are arranged in parallelogram geometry as shown in Fig. 4a. The resultant images are shown in Figs. 4b \& 4c. Due to high MTRRC and MTCRRC image obtained by narrow angle approximation is blurred. Shannon Reconstruction created a high quality focused image with this geometry of target arrangements.

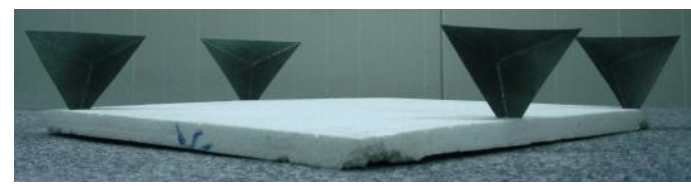

(a) Test 2 setup

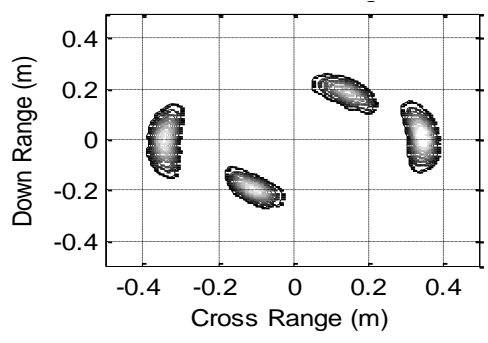

(b) Unfocused image

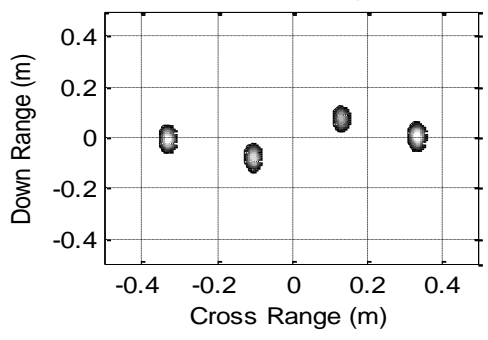

(c) Focused image

Fig. 4 Four Trihedral Corner Reflectors

\section{Test 3: Five Trihedral Corner Reflectors}

Five trihedral corner reflectors arrangement is shown in Fig.5a. The resultant images are shown in Figs.5b \& 5c. In addition to high MTRRC and MTCRRC, reflections obtained from Corner Reflectors CR3 \& C4 are weakened due to shadowing effects produced by CR1 \& CR2 respectively. Image obtained by narrow angle approximation is blurred and distorted. However, Shannon Reconstruction created a good quality focused image with this geometry of target arrangements but method could not remove the shadowing effect completely. 


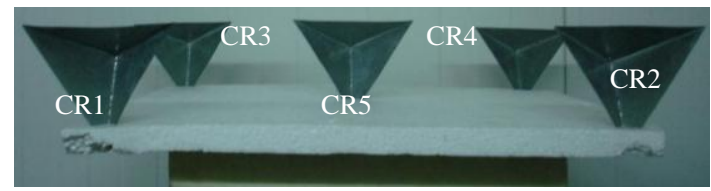

(a) Test 3 setup

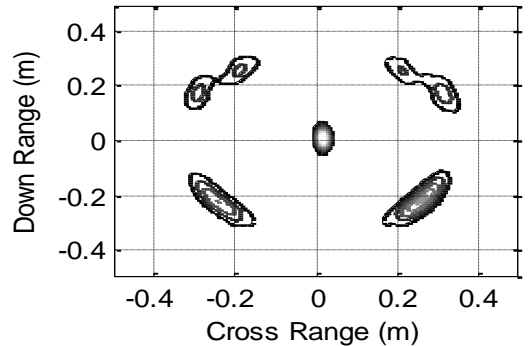

(b) Unfocused image

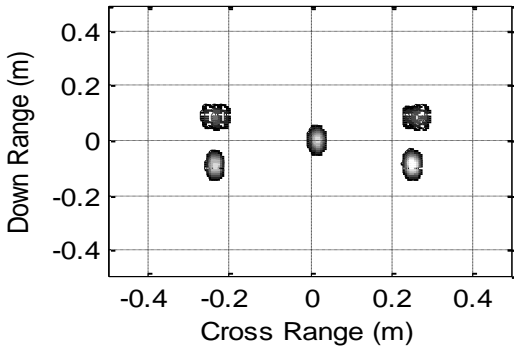

(c) Focused image

Fig.5. Five Trihedral Corner Reflectors

\section{Conclusion}

A method for focusing ISAR images based on polar reformatting technique using Shannon reconstruction is presented. Algorithm is applied on experimental data obtained from different target geometries in the Far Field RCS measurement Compact Range. In test1, where MTRC was negligible, both narrow band approximation and Polar reformatting approach gave similar results. In test2, the blurring effect produced by MTRC phenomenon for the four trihedral corner reflectors, CRs, (arranged in Parallelogram geometry) was fully corrected and highly focused by Polar reformatting. However, the blurring effect of corner reflectors (CR3 \& CR4) was partially corrected by polar reformatting due to weak reflections received from the reflectors with severe MTRC (30 cells). This happened due to the signal shadowing in target geometry, as the corner reflectors (CR3 \& CR4) were in same cross range cells behind the $\mathrm{CR} 1 \& \mathrm{CR} 2$ respectively.

\section{Acknowledgements}

The authors are especially grateful to Syed Khurram Naqib and Compact Range staff for conducting experiments and providing data for our work.

\section{References}

[1] B. Haywood, R. Kyprianou, C. Fantarella, J. McCarthy, "ISARLAB - Inverse Synthetic Aperture Radar Simulation and Processing Tool," Surveillance Systems Division Electronics and Surveillance Research Laboratory, June 1999.

[2] A. Wessling, "Radar Target Modelling Based on RCS Measurements," ISRN LITHISY-EX-3225-2002, Linköping, 2002.

[3] L. Zhang, Y. Li, M. Xin, B. Zheng; "Approach for Airborne Radar ISAR Imaging of Ship Target," IET International Radar Conference, China, 2009.

[4] D. L. Mensa, High Resolution Radar Cross-Section Imaging, Artech House, Ch. 5, 1991 
[5] B.V. Nuenen, ARCS ISAR imaging user manual, March Microwave Systems, Netherlands, ARCS Version 1.6P, 1999.

[6] E. D. Benta, "Limitations on SAR Image Area due to Motion through Resolution Cells," IEEE Trans on Aerospace and Electronic Systems, Vol. 22, No. 6, p.p. 799-803, 1986.

[7] G.Y.Lu , Z. Bao, "Compensation of Scatterer Migration through Resolution Cell in Inverse Synthetic Aperture Radar Imaging”, IEE Proceedings on Radar, Sonar and Navigation, Vol. 147, No. 2, p.p. 80-58, 2000.

[8] M. Soumekh, Synthetic Aperture Radar Signal Processing with MATLAB Algorithms, Wiley \& Sons, Inc. 1999.

[9] J. S. Son, G. Thomas, B. C. Flores, Range-Doppler Radar Imaging and Motion Compensation, Artech House, Boston, London, 2000. 